CHARACTERIZING THE LOW-MASS MOLECULAR COMPONENT IN THE NORTHERN SMALL MAGELLANIC CLOUD

This article has been downloaded from IOPscience. Please scroll down to see the full text article. 2010 ApJ 7121248

(http://iopscience.iop.org/0004-637X/712/2/1248)

The Table of Contents and more related content is available

Download details:

IP Address: 131.215.193.213

The article was downloaded on 26/03/2010 at 21:27

Please note that terms and conditions apply. 


\title{
CHARACTERIZING THE LOW-MASS MOLECULAR COMPONENT IN THE NORTHERN SMALL MAGELLANIC CLOUD
}

\author{
E. Muller ${ }^{1,2,12}$, J. Otт ${ }^{3,4,13}$, A. Hughes ${ }^{2,5}$, J. L. Pineda ${ }^{6,14}$, T. Wong ${ }^{2,7,8}$, N. Mizuno ${ }^{1,9}$, A. Kawamura ${ }^{1}$, Y. Mizuno ${ }^{1}$, \\ Y. FuKUi ${ }^{1}$, T. ONISHI ${ }^{1,10}$, AND M. Rubio ${ }^{11}$ \\ ${ }^{1}$ Department of Astrophysics, Nagoya University, Furo-cho, Chikusa-ku, Nagoya 464-8602, Japan \\ ${ }^{2}$ Australia Telescope National Facility, CSIRO, P.O. Box 76, Epping, NSW 1710, Australia \\ ${ }^{3}$ National Radio Astronomy Observatory, P.O. Box O, 1003 Lopezville Road, Socorro, NM 87801, USA \\ ${ }^{4}$ California Institute of Technology, 1200 E. California Blvd, Caltech Astronomy, 105-24, Pasadena, CA 91125, USA \\ ${ }^{5}$ Centre for Supercomputing and Astrophysics, Swinburne University of Technology, Hawthorn, VIC 3122, Australia \\ ${ }^{6}$ Jet Propulsion Laboratory, California Institute of Technology, 4800 Oak Grove Drive, Pasadena, CA 91109, USA \\ ${ }^{7}$ School of Physics, University of New South Wales, Sydney, NSW 2052, Australia \\ ${ }^{8}$ Department of Astronomy, University of Illinois, Urbana, IL 61801, USA \\ ${ }^{9}$ National Astronomical Observatory of Japan, 2-21-1 Oswa, Mitaka, Tokyo 181-8588, Japan \\ ${ }^{10}$ Department of Physical Science, Osaka Prefecture University, Gakuen 1-1, Sakai, Osaka 599-8531, Japan \\ ${ }^{11}$ Departamento de Astronomia, Universidad de Chile, Casilla 36-D, Santiago, Chile \\ Received 2009 June 5; accepted 2010 January 26; published 2010 March 12
}

\begin{abstract}
We present here the first results from a high-resolution survey of the ${ }^{12} \mathrm{CO}(J=1-0)$ emission across the northern part of the poorly enriched Small Magellanic Cloud (SMC), made with the ATNF Mopra telescope. Three molecular complexes detected in the lower resolution NANTEN survey are mapped with a beam FWHM of $\sim 42^{\prime \prime}$, to sensitivities of approximately $210 \mathrm{mK}$ per $0.9 \mathrm{~km} \mathrm{~s}^{-1}$ channel, resolving each complex into 4-7 small clouds of masses in the range of $M_{\mathrm{vir}} \sim 10^{3}-10^{4} M_{\odot}$ and with radii no larger than $16 \mathrm{pc}$. The northern SMC CO clouds follow similar empirical relationships to the southern SMC population, yet they appear relatively under-luminous for their size, suggesting that the star-forming environment in the SMC is not homogeneous. Our data also suggests that the $\mathrm{CO}$ cloud population has little or no extended $\mathrm{CO}$ envelope on scales $\gtrsim 30 \mathrm{pc}$, further evidence that the weak CO component in the north SMC is being disassociated by penetrating UV radiation. The new highresolution data provide evidence for a variable correlation of the $\mathrm{CO}$ integrated brightness with integrated $\mathrm{H}$ I and $160 \mu \mathrm{m}$ emission; in particular CO is often, but not always, found coincident with peaks of $160 \mu \mathrm{m}$ emission, verifying the need for matching-resolution $160 \mu \mathrm{m}$ and $\mathrm{H}$ I data for a complete assessment of the $\mathrm{SMC} \mathrm{H}_{2}$ mass.
\end{abstract}

Key words: galaxies: dwarf - ISM: molecules - Magellanic Clouds - radio lines: galaxies - stars: evolution

Online-only material: color figures

\section{INTRODUCTION}

Molecular clouds are associated with the very earliest stages of star formation and the environment external to the molecular cloud (e.g., enrichment levels, interstellar medium (ISM) density variations, ambient magnetic and UV fields) must also play a significant role in the star formation process. To understand the extent these and other parameters affect star formation, we must examine the evolution of molecular clouds within a range of different environmental conditions.

The Magellanic Clouds are relatively metal poor; the Large Magellanic Cloud (LMC) has a metallicity that is $~ 30 \%$ of the solar value and the Small Magellanic Cloud (SMC) about $10 \%$ of the solar value (e.g., Larsen et al. 2000). Furthermore, the molecular cloud population in the Magellanic Clouds is unique in the extra-galactic molecular cloud ensemble; Bolatto et al. (2008) show that the molecular clouds in the southwest of the SMC are anomalously weak and under-luminous in comparison with other nearby extra-galactic systems. At a distance of $\sim 60 \mathrm{kpc}$ (e.g., Cioni et al. 2000, who report $D_{\text {SMC }} \sim 63 \mathrm{kpc}$, however we use $D_{\mathrm{SMC}} \sim 60 \mathrm{kpc}$ for consistency with existing

\footnotetext{
12 Bolton Fellow, ATNF.

13 Jansky Fellow of the National Radio Astronomy Observatory. The National Radio Astronomy Observatory is a facility of the National Science Foundation operated under cooperative agreement by Associated Universities, Inc.

${ }^{14}$ NASA Postdoctoral Program Fellow.
}

literature), the SMC hosts a unique laboratory with an unenriched ISM that is similar to that found in early-epoch and unevolved galaxies.

The molecular (i.e., $\mathrm{H}_{2}$ ) component of galactic systems is most commonly estimated indirectly from observations of other tracers: e.g., from FIR or millimeter/submillimeter data sets and related to the $\mathrm{CO}$ fraction via empirically derived relationships. Leroy et al. (2007) recently combined H I and $160 \mu \mathrm{m}$ observations of the SMC to estimate the molecular distribution and the $X_{\mathrm{SMC}}$ factor (where the $X$ factor is an empirical conversion between $\mathrm{CO}$ and $\mathrm{H}_{2} ; X=N\left(\mathrm{H}_{2}\right) / W_{\mathrm{CO}}$ $\left.\mathrm{cm}^{-2}\left(\mathrm{~K} \mathrm{~km} \mathrm{~s}^{-1}\right)^{-1}\right)$. They find an $X_{\mathrm{SMC}}$ factor that is broadly in agreement with those derived from virial mass estimates, if slightly higher. A higher $X_{\mathrm{SMC}}$ factor implies that the actual mass of the $\mathrm{CO}$ clouds is greater than their measured virial mass, which may then require some magnetic support to prevent complete fragmentation. (Leroy et al. 2007; see also Bot et al. 2007 and Israel et al. 2003-hereafter SKP-X, to indicate paper $X$ of the SEST key project) go on to suggest that the $\mathrm{H}_{2}$ component, which provides more effective self-shielding than does CO, resides in larger and weaker extended envelopes and is not as effectively traced by strong $\mathrm{CO}$ emission. In this scenario, the lower dust-to-gas ratio allows UV radiation to penetrate further into the ISM and disassociate the outer CO layers to a level that is not detectable by current observations (see also Pak et al. 1998). 
However, Pineda et al. (2009) made a study of the $X$ factor as a function of a strongly varying UV field within the LMC (nearby to the bright star-forming region, 30 Doradus) and found that the value of $X$ and has a mean value of $3.9 \times 10^{20} \mathrm{~cm}^{-2}(\mathrm{~K} \mathrm{~km}$ $\left.\mathrm{s}^{-1}\right)^{-1} ; \sim 2 X_{\mathrm{Gal}}$ but does not vary with the strength of the UV field. Given the apparent lower metallicity of the SMC relative to the LMC (e.g., Rolleston et al. 2003, 2002), we expect a higher $X_{\mathrm{SMC}}$ factor and indeed, $X_{\mathrm{SMC}}$ estimates are of the order of $10^{21}$ (e.g., Leroy et al. 2007; Rubio et al. 1991; Israel et al. 1993 - hereafter SKP-I,), with some exceptions where $X_{\text {SMC }}$ is found to be $\sim 10^{20}$ (e.g., SKP-X).

Generally, however, the existing measurements of the global molecular component of the SMC have been, by virtue of the weak emission, confined to very specific regions, often comprising a single pointing (e.g., Rubio et al. 1996, 2000; Israel et al. 1993, 2003, and references therein). In particular, Rubio et al. (1993b; hereafter SKP-II) demonstrated the importance of high-resolution observations in resolving the molecular clouds and refining their basic morphological properties.

The NANTEN telescope has mapped the largest area of the SMC at any molecular transition (Mizuno et al. 2001; Blitz et al. 2007), covering the SMC bar, and parts of the eastern side. This 4 m telescope has a resolution of $\sim 2$ '. 6 at the ${ }^{12} \mathrm{CO}(J=1-0)$ line (subtending $44 \mathrm{pc}$ at $D \sim 60 \mathrm{kpc}$ ), and so despite the excellent coverage, there remain some questions regarding the extent to which NANTEN can resolve molecular clouds in the SMC.

The existing targeted studies cannot form a resolved and comprehensive statistical study of the general molecular component in the SMC. It is, therefore, still impossible to completely understand the properties of the molecular clouds in the SMC as an ensemble, or to understand any large-scale systematic variations of the molecular cloud population throughout the SMC. It is with these issues in mind that we have undertaken to map the molecular component in the northern part of the SMC at high spatial resolution $\left(\sim 42^{\prime \prime}\right)$. This project forms the SMC branch of the molecular survey of the Magellanic System: "Magellanic Mopra Assessment" (MAGMA; Ott et al. 2008; Pineda et al. 2009).

In Section 2, we detail the observations and data reduction. In Section 3, we discuss the methods used to process and analyze the resulting data cubes. In Section 4, we briefly report on the results. Section 5 contains a discussion of the detected CO population in the context of the SMC ISM; and Section 6 summarizes our findings.

\section{OBSERVATIONS AND DATA REDUCTION}

Observation targets were selected from clouds in the north of the SMC that showed an integrated CO intensity of at least $0.2 \mathrm{~K} \mathrm{~km} \mathrm{~s}^{-1}$ (as measured by NANTEN; Mizuno et al. 2001). These clouds include those labeled NE-1 and NE-3; mapped at 8'.8 by Rubio et al. (1991), and we further extend the ensemble to include a previously unlabeled cloud, which we call NE-4. NE-4 is visible in maps by NANTEN (Mizuno et al. 2001) but has no specific reference in that work.

Observations were conducted during 2007, August and September with the Mopra $22 \mathrm{~m}^{\text {Telescope }}{ }^{15}$ in Australia, and

\footnotetext{
15 The Mopra radio telescope is part of the Australia Telescope which is funded by the Commonwealth of Australia for operation as a National Facility managed by CSIRO. The University of New South Wales Digital filter bank used for the observations with the Mopra Telescope was provided with support from the Australian Research Council.
}

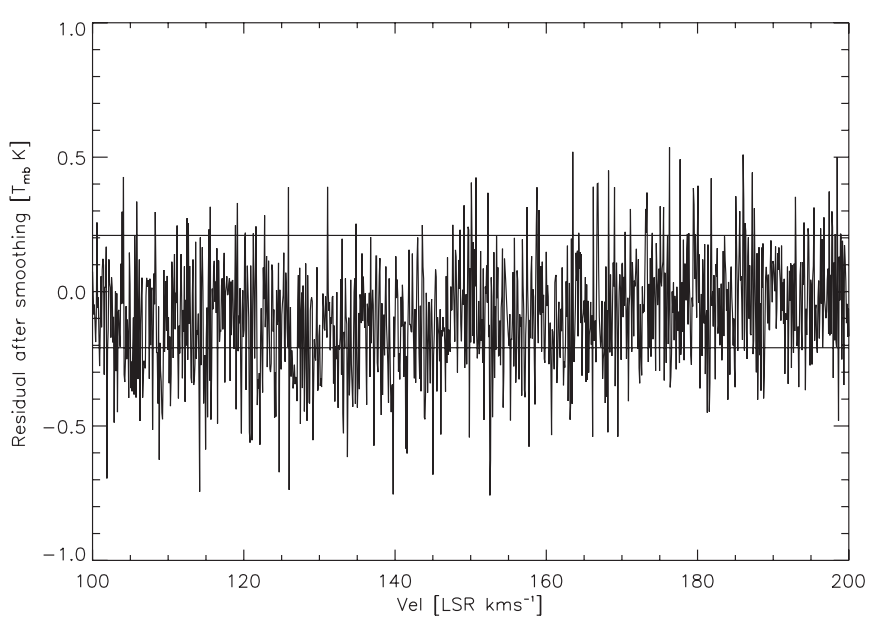

Figure 1. Example of component that is removed from a sightline, following the spatial frequency-domain smoothing process described in the text. This spectrum is toward NE-1a $(00: 59: 45-71: 44: 40 ; \mathrm{J} 2000)$. The two horizontal lines indicate $\pm 1 \sigma$ levels.

the UNSW-MOPS spectrometer. ${ }^{16}$ Observations were made using the "zoom" mode, where the $138 \mathrm{MHz}$-wide band is sampled with 4096 channels. Pointing accuracy was maintained by frequent (every $1.5 \mathrm{hr}$ ) observations of the $\mathrm{SiO}$ maser Upsilon Mensa, so that errors were typically less than $5^{\prime \prime}$. Calibration and measurements of the $T_{\text {sys }}$ were made from frequent ( $\sim 15$ minute interval) measurements of a hot load. The system temperature and data quality were monitored and calibrated using an in-horn diode calibrator.

Areas were scanned in overlapping $5^{\prime} \times 5^{\prime}$ regions. Each area was observed twice with a sampling rate of $2 \mathrm{~s}$ (equivalent to three integrations per $\sim 30^{\prime \prime}$ telescope beam-width), with orthogonal scanning directions to reduce scanning artifacts. Observations of ${ }^{12} \mathrm{CO}(1-0)$ in Orion $\mathrm{KL}$ were made once each observing session to monitor telescope stability. The resulting peak flux measurements were found to be self-consistent within $10 \%$, and also with observations made by the SEST telescope ${ }^{17}$ (see also Ladd et al. 2005). The data were baseline-corrected with a zeroth order polynomial fit and doppler-corrected using the AIPS++ LIVEDATA package.

The cubes were formed using median weighting with the GRIDZILLA package (beam-weighted gridding, and further smoothed with a radius-truncated Gaussian kernel, both with a FWHM of $\sim 35$ arcsec). Some of the spectra have a low-order and low-amplitude baseline variation present, which causes spurious detections in analysis software used later in this study. To remove this low-order baseline, the data cubes were differenced with a duplicate data cube, which was heavily smoothed in the frequency domain (width $=26 \mathrm{~km} \mathrm{~s}^{-1}$ ). As the expected line widths of the $\mathrm{CO}$ emission were of the order of a few $\mathrm{km} \mathrm{s}^{-1}$ (e.g., Rubio et al. 1996), this step does not significantly affect the measurement and results in a relatively minor adjustment to the mean power per channel (which was always less than the $1 \sigma$ noise level, as shown in Figure 1). Also note that any systematic or random errors arising from the baseline correction made here have not propagated through to the final brightness temperature estimates. Making this spectral smoothing-and-subtraction

\footnotetext{
16 The University of New South Wales Digital Filter Bank used for the observations with the Mopra Telescope was provided with support from the Australian Research Council.

17 http://www.apex-telescope.org/sest/html/telescope-calibration /calib-sources/orionkl.html
} 
step improved the reliability of the cloud-searching algorithm by forcing the mean power outside channels containing emission to be approximately zero, and reduces false detections associated with a slowly varying spectral baseline.

Finally, to improve sensitivity, the data cubes were binned to $0.9 \mathrm{~km} \mathrm{~s}^{-1}$ and convolved with $30^{\prime \prime}$ kernel, yielding an effective resolution of $42^{\prime \prime}(\sim 12 \mathrm{pc})$. The data cube was formed with a pixel size of $15^{\prime \prime}(\sim 4 \mathrm{pc})$. It should be noted that MOPS uses a large digital filter bank, rather than a traditional autocorrelator that operates in the Fourier domain. As a result, the frequency resolution is well approximated by the frequency channel spacing. Assuming an main-beam efficiency of 0.47 (e.g., Hughes et al. 2010) at $115 \mathrm{GHz}$ for the Mopra telescope, the resulting rms level per channel is $\sim 210 \mathrm{mK}\left(T_{\mathrm{mb}}\right)$.

The maps and spectra for regions NE-1, NE-3, and NE-4 are shown in Figures 2-4. These figures show the integrated intensity for pixels that were detected by CLOUDPROPS as containing emission only (See Section 3).

\section{CLOUD IDENTIFICATION PROCEDURE}

The literature contains various methods of determining the radius of CO clouds detected in the SMC. Rubio et al. (1991) and Mizuno et al. (2001) choose to define the extent of the cloud based on the sensitivity of the data: pixels brighter than $3 \sigma$ in the integrated intensity maps are added and represent the area of the cloud. We attempt here an approach that is slightly less dependent on the quality of the observational data, where clusters of spectral lines containing emission are detected using a moment analysis by the (Rosolowsky \& Leroy 2006) analysis package CLOUDPROPS. We stress that CLOUDPROPS is used here only to objectively identify spectra containing emission within four adjacent velocity channels. CLOUDPROPS is not used for further analysis or interpretation and the observable parameters are measured from pixels that are identified by CLOUDPROPS as containing emission.

We apply CLOUDPROPS to identify regions containing signal greater than $1.5 \sigma$ (by setting the thresh parameter) over at least four spectral channels and spread over an area equal to or greater than the beam size $\left(\sim 42^{\prime \prime}\right)$. We further examine cloud candidates by eye to eliminate spurious or marginal identifications. Typically, one or two putative "clouds" in each of the three regions were falsely identified by CLOUDPROPS, and were rejected.

We estimate the cloud radii, $I_{\mathrm{CO}}$ and $L_{\mathrm{CO}}$ by summing the area of pixels containing emission that are close to the same local integrated intensity maxima (i.e., we group together all pixels that are closest to the same local maxima). We use the common definitions of these parameters: radius, $R=(\text { Area } / \pi)^{0.5}$, virial mass; where $M_{\mathrm{VIR}}=190 R \Delta V^{2}$ (assuming a $1 / r$ density profile throughout the cloud and where the velocity width $(\Delta V)$ is measured at the position of maximum integrated brightness); and $\mathrm{CO}$ luminosity $L_{\mathrm{CO}}$, estimated from the product of the mean integrated emission and cloud area. As the Mopra beam size and shape is not well understood and the SMC cloud radii are closely matched to the Mopra beam size, we do not present the de-convolved radii of the clouds. We instead incorporate the deconvolved radius in the measurement errors by calculating the upper and lower errors separately: the lower error is equal to the de-convolved radius minus the measurement error of $2 \mathrm{pc}$. The errors reported for peak temperature are measurement errors only: we do not attempt to propagate the errors of the radius measurements and recalculate peak temperature measurement errors arising from beam dilution.
Line characteristics of spectra toward the position of peakintegrated brightness (main-beam brightness temperature, $T_{\mathrm{mb}}$; velocity width, $\Delta V$; velocity centroid, $V_{0}$ ) are estimated by fitting a single-component Gaussian (using the MarquardtLevenberg algorithm). Given the low signal-to-noise ratio $(\mathrm{S} / \mathrm{N})$ of these CO spectra and their simple, single-component profiles, it is not appropriate or beneficial to seek to decompose the emission into more than one spectral component. The errors for observables obtained in this way are estimated as asymptotic errors using statistical algorithms developed by Landman et al. (1982).

It should be noted that the low $\mathrm{S} / \mathrm{N}$ of these observations propagates to large errors in the fitted parameters, typically of around $50 \%$ or even greater. As such, the resulting best-fit Gaussian profiles were occasionally narrower than the instrumental resolution of the telescope (e.g., cloud NE-3d). Table 1 lists the cloud parameters : Peak $T_{\mathrm{mb}}, V_{0}, \Delta V$, radius $R, M_{\mathrm{VIR}}, I_{\mathrm{CO}}$.

To provide some assurance of the effectiveness of our data preparation and analysis technique, we apply it also to data of the southwest SMC, taken with the SEST telescope (Rubio et al. 1993a, hereafter, SKP-III). The analysis method we apply to the SEST data set is almost identical to that used on the data presented here, with the exception of the smoothing-andsubtraction baseline correction step. This step was omitted primarily because the frequency-switching mode employed for the SEST observations generates a "negative" spectral artifact nearby to the real emission spectrum, and also because insufficient line-free channels exist to create a usable smoothing function (as the emission line occurs near the edge of the band). Instead, we have removed a second-order baseline, interpolated from line-free channels across velocities channels where emission occurs.

We provide the results of this comparison in Table 2 and show that our method produces values that are consistent to within approximately $10 \%$ with that reported in SKP-III. Note that errors are not quoted in SKP-II and SKP-III, however the rms for the SKP observations is cited as $200 \mathrm{mK}$, and later papers in that series cite $T_{\mathrm{mb}}$ accuracies of $15 \%-20 \%$.

\section{PROPERTIES OF CO CLOUDS IN THE SMC}

Table 1 shows that the detected and most massive clouds in the northern SMC are relatively small, with virial masses in the $10^{3}-10^{4} M_{\odot}$ range. This is at the extreme low end of an extra-galactic sample examined by Bolatto et al. (2008), where the virial masses of the extra-galactic molecular cloud ensemble exist over a range of $\sim 10^{4}-10^{6} M_{\odot}$. We see then that the molecular cloud population in the northern SMC can be regarded as an extreme population relative to the southwest SMC, and more so in the extra-galactic context.

Figures 2-4 show the relative distributions and ${ }^{12} \mathrm{CO}(J=1-$ $0)$ emission spectra of the three regions NE-1, NE-3, and NE-4. Also shown are the velocity-integrated $I_{\mathrm{CO}}$ maps overlaid on $160 \mu \mathrm{m}$ and $\mathrm{H} \mathrm{I}$ data, as tracers of the big-grain (BG) dust abundance and temperature, and the ubiquitous ISM, respectively. Note that the H I maps are of much poorer resolution $\left(\sim 98^{\prime \prime}\right)$ than the $\mathrm{CO}$ data set and are used here to aid a contextual discussion of the relationships between $\mathrm{HI}, \mathrm{CO}$, and $160 \mu \mathrm{m}$ tracers. The $160 \mu \mathrm{m}$ data is obtained directly from the $\mathrm{S}^{3} \mathrm{MC}$ archive (Bolatto et al. 2007) ${ }^{18}$ and have not been rigorously processed to remove, for example, the weak (but very complicated) contribution from foreground cirrus (see Leroy et al. 2007).

\footnotetext{
18 http://celestial.berkeley.edu/spitzer/
} 

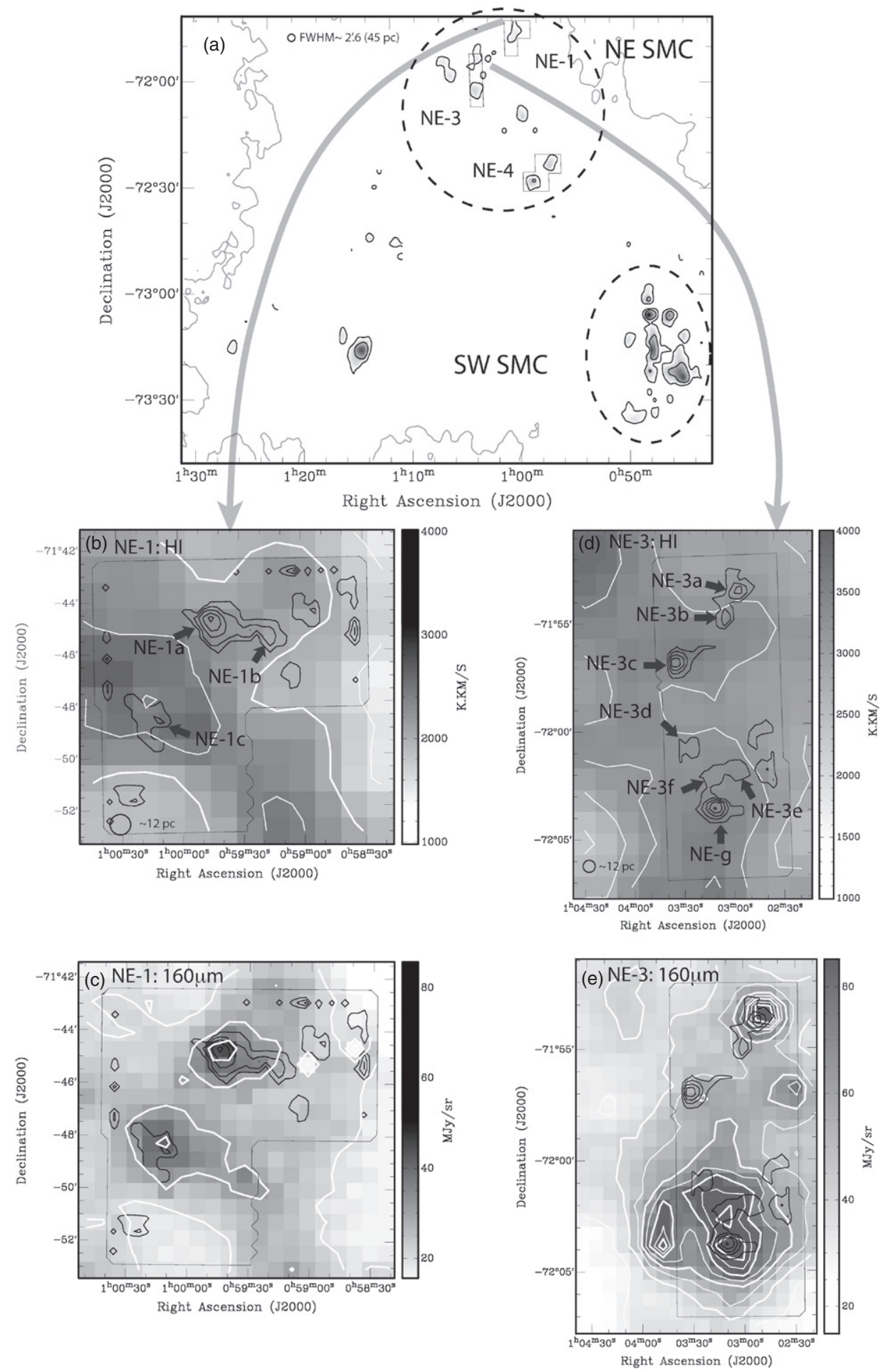

Figure 2. Panel (a): map of ${ }^{12} \mathrm{CO}(1-0)$, from Mizuno et al. (2001), with boxes locating the areas which were observed by Mopra. Black contours are 0.3 , $0.9 \mathrm{~K} \mathrm{~km} \mathrm{~s}^{-1}$. NE-1 and NE-3 are shown in panels (c) and (d), see Figure 4 for NE-4. The dotted ellipses clarify the terminology for "northeast" and "southwest" regions, as used in this report. The gray outer contour shows the $1 \times 10^{21} \mathrm{H}$ I integrated intensity level. Panels (b) and (c): contours of Mopra CO observations (black) made around NE-1, overlaid on integrated H I (b) (Stanimirovic 1999) and $160 \mu \mathrm{m}$ (c) observations (Bolatto et al. 2007). CO contours show integrated brightness temperature levels $0.5+1 \mathrm{~K} \mathrm{~km} \mathrm{~s}^{-1}$; white $\mathrm{HI}$ and $160 \mu \mathrm{m}$ contours are to aid visualization, and are at intervals of $400 \mathrm{~K} \mathrm{~km} \mathrm{~s}^{-1}$ and $10 \mathrm{MJy}$ Str ${ }^{-1}$, respectively. Panels (d) and (e): contours of Mopra CO observations made around NE-3, overlaid on integrated HI (d) and $160 \mu \mathrm{m}$ (e) observations. CO contours show the $0.5+1 \mathrm{~K} \mathrm{~km} \mathrm{~s}^{-1}$; H I and $160 \mu \mathrm{m}$ contours are to aid visualization, and are at intervals of $400 \mathrm{~K} \mathrm{~km} \mathrm{~s}^{-1}$ and $10 \mathrm{MJy} \mathrm{Str}^{-1}$, respectively.

\subsection{NE-1}

We see in Figure 2 (panels (b) and (c)) the spatial relationship of NE-1 with $\mathrm{HI}$ and $160 \mu \mathrm{m}$, respectively. The CO emission from this region is relatively weak, with a peak $T_{\mathrm{mb}}$ of barely over $1 \mathrm{~K}$. The detected $\mathrm{CO}$ clouds are spatially coincident with the strongest sources of $160 \mu \mathrm{m}$ emission in this region (Figure 2(b)), although there are some significant departures from a perfect correlation, most likely due to varying dust temperature and/or small-scale fluctuations in H I column den- 

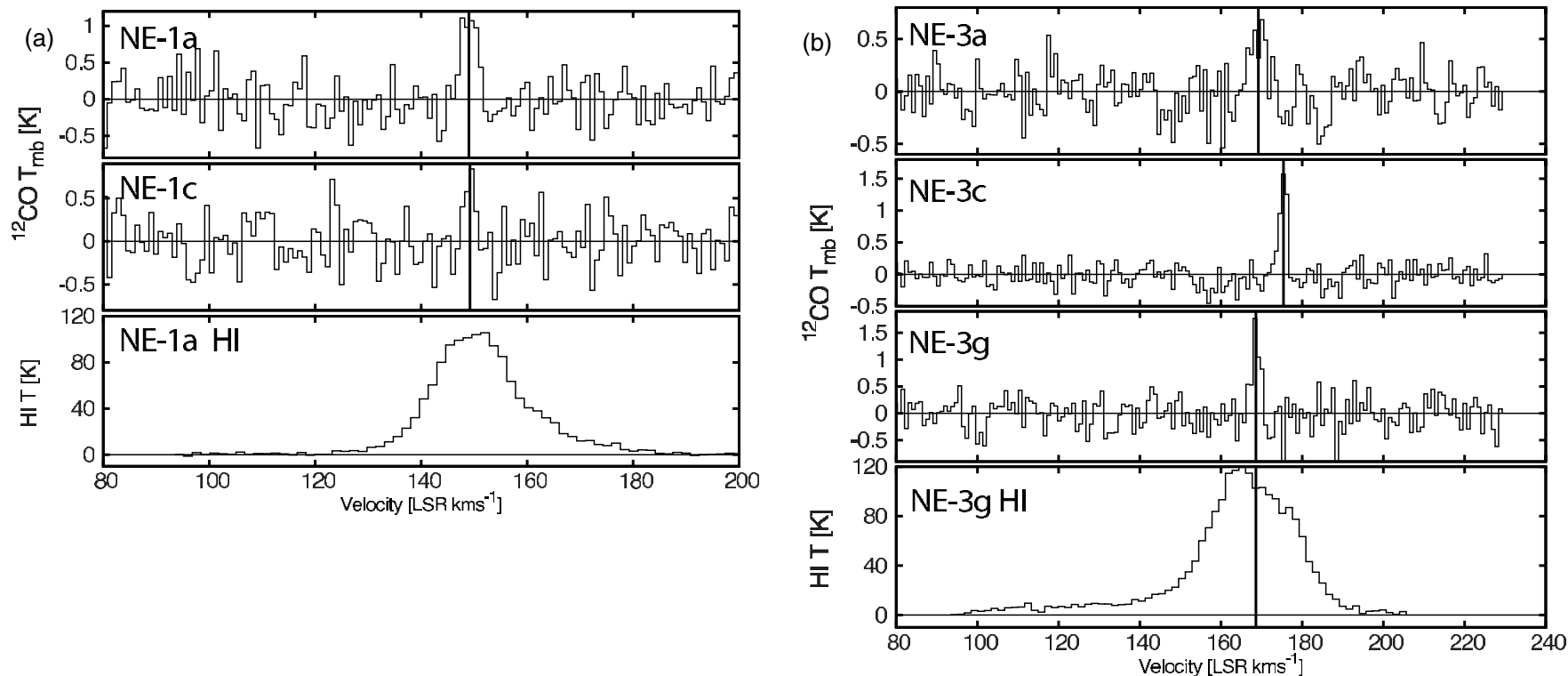

Figure 3. Panels (a): CO spectra toward peak positions of NE-1a and NE-1c, and H I spectra toward NE-1a bottom. The velocity centroids are marked with a vertical line. Note that the H I profile shown in the bottom is measured toward NE-1a and approximately the same throughout the entire of NE-1. Panels (b): CO spectra toward peak positions of NE-3a, NE-3c and NE-3g, and $\mathrm{H}$ I spectra toward NE-3g bottom. The CO velocity centroids are marked with a vertical line. As for panel (b), the $\mathrm{H} \mathrm{I}$ profile is measured only toward NE-3g, but is approximately the same throughout the entire of NE-3.

Table 1

Northern SMC CO Cloud Parameters

\begin{tabular}{|c|c|c|c|c|c|c|c|c|}
\hline Cloud/Complex & $\begin{array}{c}\text { R.A. } \\
(\text { J2000) }\end{array}$ & $\begin{array}{c}\text { Decl. } \\
(\mathrm{J} 2000)\end{array}$ & $\begin{array}{c}\text { Peak } T_{\mathrm{mb}} \\
(\mathrm{K})\end{array}$ & $\begin{array}{c}V_{o} \\
\left(\mathrm{~km} \mathrm{~s}^{-1}\right)\end{array}$ & $\begin{array}{c}\Delta V \\
\left(\mathrm{~km} \mathrm{~s}^{-1}\right)\end{array}$ & $\begin{array}{c}\text { Radius }^{\mathrm{a}} \\
(\mathrm{pc})\end{array}$ & $\begin{array}{c}M_{\mathrm{VIR}} \\
\left(\times 10^{3} M_{\odot}\right) \\
\end{array}$ & $\begin{array}{c}L_{\mathrm{CO}} \times 10^{3} \\
\left(\mathrm{~K} \mathrm{~km} \mathrm{~s}^{-1} \mathrm{pc}^{2}\right)\end{array}$ \\
\hline \multicolumn{9}{|l|}{ Complex NE-1 } \\
\hline NE-1a & $00: 59: 50$ & $-71: 44: 40$ & $1.2 \pm 0.2$ & $149.0 \pm 0.2$ & $3.4 \pm 0.6$ & $16_{-3}^{+2}$ & $40_{-10}^{+10}$ & $1.5_{-0.7}^{+0.5}$ \\
\hline$N E-1 b$ & $00: 59: 20$ & $-71: 45: 30$ & $0.8 \pm 0.3$ & $149.2 \pm 0.3$ & $2.1 \pm 0.8$ & $9_{-5}^{+2}$ & $8_{-7}^{+6}$ & $0.3_{-0.3}^{+0.2}$ \\
\hline NE-1c & 01:00:10 & $-71: 48: 40$ & $0.9 \pm 0.4$ & $150.0 \pm 0.3$ & $2.0 \pm 0.7$ & $13_{-3}^{+2}$ & $10_{-8}^{+7}$ & $0.6_{-0.3}^{+0.2}$ \\
\hline \multicolumn{9}{|c|}{ Complex NE-3/N76 } \\
\hline NE-3a & 01:02:50 & $-71: 53: 30$ & $0.6 \pm 0.1$ & $169.2 \pm 0.5$ & $5 \pm 1$ & $12_{-3}^{+2}$ & $60_{-30}^{+30}$ & $0.6_{-0.4}^{+0.3}$ \\
\hline$N E-3 b$ & 01:03:00 & $-71: 55: 00$ & $0.7 \pm 0.2$ & $171.9 \pm 0.3$ & $2.4 \pm 0.6$ & $9_{-4}^{+2}$ & $11_{-8}^{+6}$ & $0.3_{-0.3}^{+0.2}$ \\
\hline $\mathrm{NE}-3 \mathrm{c}$ & 01:03:30 & $-71: 57: 00$ & $1.7 \pm 0.2$ & $175.4 \pm 0.1$ & $2.1 \pm 0.3$ & $11_{-4}^{+2}$ & $10_{-4}^{+3}$ & $0.7_{-0.5}^{+0.3}$ \\
\hline NE-3d & 01:03:20 & $-72: 01: 00$ & $0.6 \pm 0.2$ & $168.6 \pm 0.2$ & $2.3 \pm 0.8$ & $7_{-7}^{+2}$ & $8_{-8}^{+6}$ & $0.2_{-0.2}^{+0.1}$ \\
\hline $\mathrm{NE}-3 \mathrm{e}$ & 01:03:00 & $-72: 02: 00$ & $0.7 \pm 0.2$ & $168.3 \pm 0.4$ & $2.4 \pm 0.6$ & $9_{-4}^{+2}$ & $11_{-8}^{+6}$ & $0.3_{-0.3}^{+0.2}$ \\
\hline NE-3f & 01:03:10 & $-72: 02: 00$ & $0.8 \pm 0.2$ & $168.1 \pm 0.2$ & $2.4 \pm 0.6$ & $9_{-4}^{+2}$ & $11_{-8}^{+6}$ & $0.3_{-0.3}^{+0.2}$ \\
\hline NE-3g & 01:03:10 & $-72: 03: 50$ & $1.6 \pm 0.3$ & $168.6 \pm 0.2$ & $2.3 \pm 0.5$ & $13_{-3}^{+2}$ & $14_{-7}^{+6}$ & $1.0_{-0.5}^{+0.3}$ \\
\hline \multicolumn{9}{|l|}{ Complex NE-4 } \\
\hline NE-4a $a_{h i}$ & 00:57:00 & $-72: 22: 40$ & $1.5 \pm 0.3$ & $153.9 \pm 0.2$ & $2.2 \pm 0.5$ & $11_{-4}^{+2}$ & $11_{-6}^{+5}$ & $0.6_{-0.4}^{+0.2}$ \\
\hline NE- $4 b_{h i}$ & $00: 56: 50$ & $-72: 24: 20$ & $0.8 \pm 0.2$ & $154.1 \pm 0.3$ & $1.8 \pm 0.6$ & $9_{-5}^{+2}$ & $6_{-5}^{+4}$ & $0.2_{-0.2}^{+0.1}$ \\
\hline NE-4c $c_{h i}$ & $00: 58: 20$ & $-72: 28: 10$ & $1.3 \pm 0.2$ & $158.2 \pm 0.2$ & $2.3 \pm 0.5$ & $15_{-3}^{+2}$ & $16_{-8}^{+7}$ & $0.9_{-0.5}^{+0.3}$ \\
\hline NE- $4 c_{\text {low }}$ & 00:58:40 & $-72: 27: 40$ & $1.6 \pm 0.3$ & $122 \pm 1$ & $5 \pm 2$ & $14_{-3}^{+2}$ & $70_{-60}^{+60}$ & $1.7_{-0.9}^{+0.5}$ \\
\hline
\end{tabular}

Note. ${ }^{\text {a }}$ Lower errors for cloud radii include potential impact smearing by the Mopra beam (FWHM $\sim 12 \mathrm{pc}$ ).

sity that is not obvious from Figure 2(c). Even so, and bearing in mind that the spatial resolution of the H I observations is $98^{\prime \prime}$; approximately 3 times that of the $\mathrm{CO}$ observations, Figure 2(c) shows the $\mathrm{H}$ I integrated brightness varies only very slowly across the region with little evidence of any significant variations in intensity toward the $\mathrm{CO}$ sources.

We estimate the virial masses of the clouds (NE-1a, NE-1b, and NE-1c) to be $(4.0 \pm 1) \times 10^{4} M_{\odot}, 8_{-7}^{+6} \times 10^{3} M_{\odot}$, and $10_{-8}^{+7} \times$ $10^{3} M_{\odot}$. Summed together, the mass of this complex measured by Mopra is approximately a factor of 50 below the virial mass estimated by Rubio et al. (1991). The combination of a lower resolved velocity dispersion (by a factor of $\sim 3$ ) and lower radius (by a factor of $\sim 6$ ) will account for the disagreements in estimated virial masses; however, we explore the wider scales of this complex further in Section 5.2.

The emission profiles associated with NE-1a and NE-1c are shown in Figure 3 (panels (a) and (b)) and these observations have enabled the velocity components of the emission regions to be separated into much narrower profiles than was previously known. We see that the $\mathrm{CO}$ peaks generally coincide with H I peaks, although not always at exactly the peak velocity. 

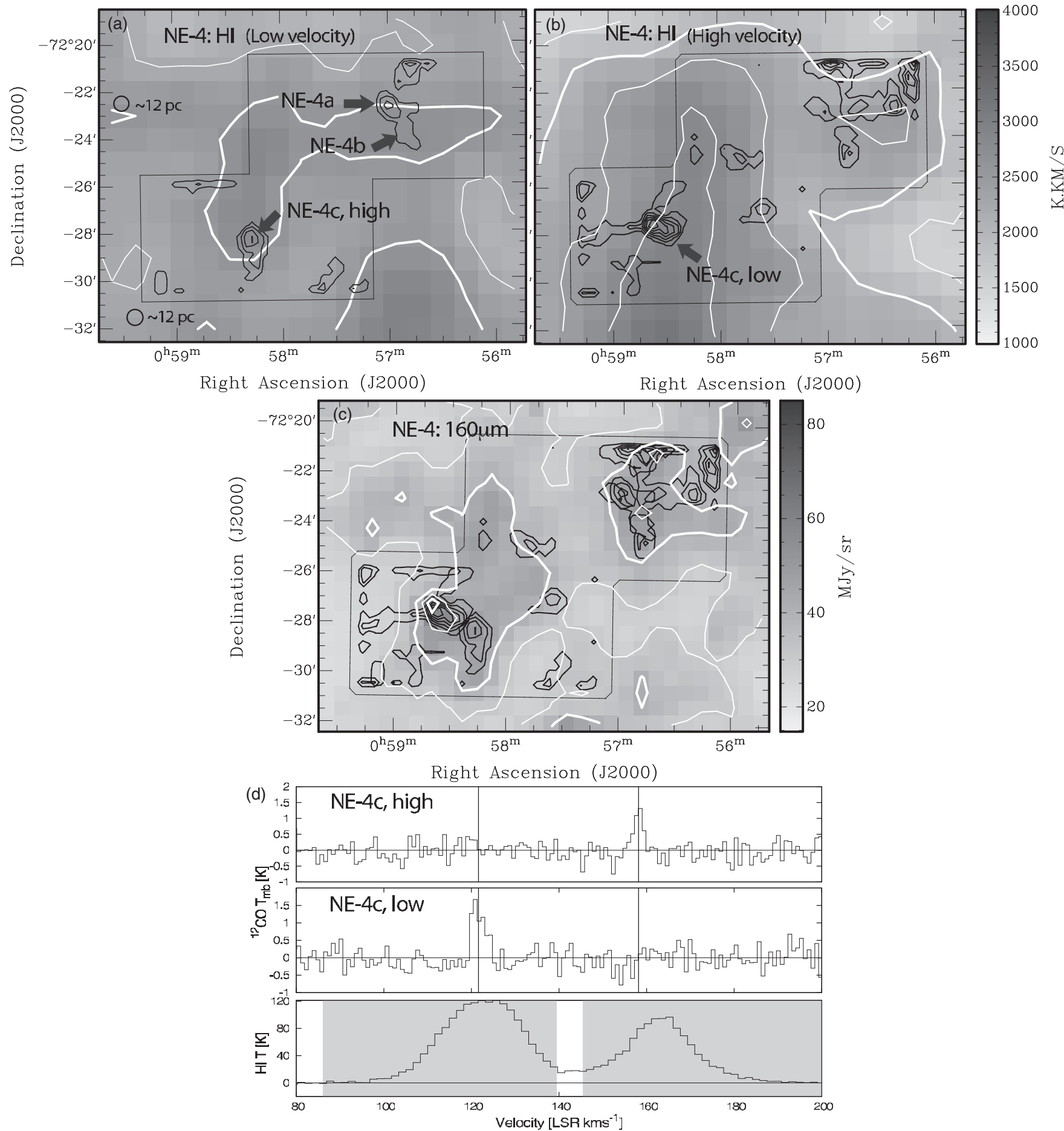

Figure 4. Contours of Mopra CO observations made around NE-4, overlaid as black contours $\left(0.5+1 \mathrm{~K} \mathrm{~km} \mathrm{~s}^{-1}\right.$ ) on integrated $\mathrm{HI}$ (panels (a) and (b); Stanimirovic 1999) and $160 \mu \mathrm{m}$ (c) observations (Bolatto et al. 2007). The CO emission is broken into the high (a) and low (b) velocity component and shown with the HI also integrated over the high- and low-velocity ranges (shown as grayed regions in the top spectra of (d). Both H I and CO components are shown together in panel (c). White contours showing the $\mathrm{HI}$ in intervals of $400 \mathrm{~K} \mathrm{~km} \mathrm{~s}^{-1}$ (in panels (a) and (b)), and in intervals of $10 \mathrm{MJy} \mathrm{str}^{-1}$ (in panel (c)) to aid visualization.

\subsection{NE-3}

The $\mathrm{CO}$ emission from this region is shown overlaid on $\mathrm{HI}$ integrated intensity and on $160 \mu \mathrm{m}$ brightness in Figure 2 (panels (d) and (e), respectively). The figures show a slightly larger area than was mapped by Mopra to include the full extent of an apparently coherent $160 \mu \mathrm{m}$ emission region. We resolve up to seven peaks of emission distributed throughout this region.
We find a total virial mass of $1.25_{-73}^{+6.3} \times 10^{5} M_{\odot}$; a factor of $\sim 100$ less than that reported by Rubio et al. (1991). The difference in measured velocity widths and radii cannot account for the difference in the estimated virial masses, however, there appears to be some differences in the CO maps from Mizuno et al. (2001) and Rubio et al. (1991). After allowing for different beam sizes, we find that the morphology of the NANTEN results is more consistent with the high-resolution results presented here. We note that the NANTEN maps show additional CO 
Table 2

Confirmation of Cloud Analysis Algorithm

\begin{tabular}{lcc}
\hline \hline \multicolumn{1}{c}{ Cloud/Object } & This Paper & SKP-II, SKP-III \\
\hline LIRS36 & & \\
$T_{\mathrm{mb}}(\mathrm{K})$ & $2.4 \pm 0.1$ & 2.37 \\
$\Delta V\left(\mathrm{~K} \mathrm{~km} \mathrm{~s}{ }^{-1}\right)$ & $3.1 \pm 0.1$ & 3.1 \\
$R(\mathrm{pc})$ & $16.3 \pm 0.2$ & 18.6 \\
$L_{\mathrm{CO}}\left(\times 10^{3} \mathrm{~K} \mathrm{~km} \mathrm{~s}^{-1} \mathrm{pc}^{2}\right)$ & $2.0 \pm 0.5$ & 2.8 \\
\hline LIRS49 & & \\
$T_{\mathrm{mb}}(\mathrm{K})$ & $1.6 \pm 0.1$ & 1.88 \\
$\Delta V\left(\mathrm{~K} \mathrm{~km} \mathrm{~s}{ }^{-1}\right)$ & $4.8 \pm 0.1$ & 4.8 \\
$R(\mathrm{pc})$ & $17.3 \pm 0.2$ & 18.6 \\
$L_{\mathrm{CO}}\left(\times 10^{3} \mathrm{~K} \mathrm{~km} \mathrm{~s}^{-1} \mathrm{pc}^{2}\right)$ & $6 \pm 1$ & 6.41 \\
\hline $\mathrm{SMCB} 1$ & & \\
$T_{\mathrm{mb}}(\mathrm{K})$ & $1.4 \pm 0.1$ & 1.38 \\
$\Delta V\left(\mathrm{~K} \mathrm{~km} \mathrm{~s}{ }^{-1}\right)$ & $2.75 \pm 0.1$ & 2.88 \\
$R(\mathrm{pc})$ & $15.2 \pm 0.2$ & 13.8 \\
$L_{\mathrm{CO}}\left(\times 10^{3} \mathrm{~K} \mathrm{~km} \mathrm{~s}^{-1} \mathrm{pc}^{2}\right)$ & $1.3 \pm 0.4$ & 1.21 \\
\hline $\mathrm{N} 88$ & & \\
$T_{\mathrm{mb}}(\mathrm{K})$ & $0.79 \pm 0.1$ & 0.77 \\
$\Delta V\left(\mathrm{~K} \mathrm{~km} \mathrm{~s}{ }^{-1}\right)$ & $1.63 \pm 0.2$ & 1.6 \\
$R(\mathrm{pc})$ & Unresolved & Unresolved \\
$L_{\mathrm{CO}}\left(\times 10^{3} \mathrm{~K} \mathrm{~km} \mathrm{~s}^{-1} \mathrm{pc}^{2}\right)$ & $0.5 \pm 0.2$ & 0.54 \\
\hline
\end{tabular}

Note. ${ }^{\text {a }}$ Note that measurement errors are not quoted in SKP-II or SKP-III; however, the $1 \sigma$ noise temperature of those observations is $\sim 0.2 \mathrm{~K}$. Note that other papers in that series (SKP-1, SKP-X) suggest a $15 \%-20 \%$ error in flux calibration. Line width $(\Delta V)$ errors for the objects above are only cited in SKP-V, which are different, but similar to the $\Delta V$ values reported in SKP-II and SKP-III. These errors quoted in SKP-V are typically $\sim 0.2 \mathrm{~km} \mathrm{~s}^{-1}$.

clouds that seem confused or absent in the Rubio et al. (1991) study, and are approximately $10^{\prime}$ east of the area mapped with Mopra for the present study. We present a study of the larger scale distribution of $\mathrm{CO}$ in the context of the NANTEN data set in Section 5.2.

The distribution of the $160 \mu \mathrm{m}$ emission associated with NE-3 again shows a generally good association of the peak $160 \mu \mathrm{m}$ and $\mathrm{CO}$ (Figure 2(e)), and there are again some notable deviations from a good correlation. The H I data (Figure 2(d)) shows a rather slowly varying and uncorrelated distribution but are not of sufficient resolution to obtain a good estimate of the relationship of $\mathrm{CO}$ and $\mathrm{H}_{2}$ on these scales observed with Mopra.

\subsection{NE-4}

The measured $\mathrm{CO}$ emission from NE-4 is shown in the top of Figure 4 as contours overlaid on $\mathrm{HI}$ integrated over $85<V_{\mathrm{LSR}}<140$ (panel (a)) and $145<V_{\mathrm{LSR}}<200$ (panel (b)). All CO integrated over the line of sight is shown overlaid on $160 \mu \mathrm{m}$ emission on panel (c) (Bolatto et al. 2007).

Studies by Mizuno et al. (2001) resolve this complex into two clouds, although this work did not discuss this region in detail. We show here in Figure 4 that this region is now resolved further into four clouds, including a pair that shows evidence for easily separable high- and low-velocity components, as shown in Figure 4(d).

Panel (d) of Figure 4 shows that the $\mathrm{CO}$ emission peaks correspond closely, although not exactly in velocity to peaks of H I. This suggests that the molecular and neutral components are to some extent, co-moving with the H I. The projected spatial separation of the high- and low-velocity components of the two CO clouds is $\sim 30 \mathrm{pc}$. The fact that two separate regions are detected in close proximity may be evidence for a recent energetic event which has bifurcated a formed molecular cloud; however, we will defer a more dedicated analysis of the velocity structure of the ISM at this location to a later paper.

The distribution of the $160 \mu \mathrm{m}$ emission shown in Figure 4(c), shows local maxima approximately spatially coincident with the $\mathrm{CO}$ emission, however once again, the resolution of the H I data (Figure 4(c)) is too poor to permit a reliable estimate of the correlation of $\mathrm{H}_{2}$ and $\mathrm{CO}$ on the $\sim 30 \mathrm{pc}$ scales of the $\mathrm{CO}$ data.

\section{ANALYSIS AND DISCUSSION}

We now have a means to examine the variations of the molecular cloud population throughout two widely separated regions in the SMC. Its dynamic and perturbed evolution suggests that different parts of the SMC may have evolved under slightly different conditions, and these new results enable a new level of analysis into the ISM of the SMC, specifically in the context of star formation in its low-metallicity environment. The compact nature of the detected CO population contrasts the relatively more extended dust emission and provides further impetus for future studies of the relationship of $\mathrm{CO}, \mathrm{HI}$, and $\mathrm{H}_{2}$. This is particularly true in the case of the low-extinction ISM of the SMC, where extended CO is more easily photodisassociated (e.g., see Maloney \& Black 1988; McKee 1989; Pak et al. 1998). However, we are now able to make some general observations regarding the distribution of small-scale $\mathrm{CO}$ and $160 \mu \mathrm{m}$ brightness - in particular reference to the $X_{\mathrm{SMC}}$ factor, and we are now in a position to examine the relationship of $\mathrm{H} \mathrm{I}$ and $\mathrm{CO}$ on $\sim 30 \mathrm{pc}$ scales in the SMC.

\subsection{Regional Variation of Molecular Cloud Properties}

In Figure 5, we compare the characteristics of the $\mathrm{CO}$ clouds measured in this study to those in the southwest SMC, made using the SEST telescope in SKP-III. It should be noted that these observations by the SEST key projects are of comparable resolution and sensitivity to our Mopra observations of the northwest clouds. Overlaid on each of the panels in the figure are empirical relationships for the plotted properties as derived in SKP-III $\left(\log \Delta V=0.51 \log R+0.04, \log L_{\mathrm{CO}}=2.17 \log \Delta V+\right.$ 2.01 , and $\left.\log M_{\mathrm{vir}}=1.46 \log L_{\mathrm{CO}}-0.09\right)$. Although the error bars for our data are large, we find that the ratios of luminosities and virial masses of the northern clouds (triangles in Figure 5, left panel) are broadly consistent with those of the southwestern population (plotted as diamonds). It is interesting that despite the substantial scatter in the points, they appear to cluster more coherently around a constant $X_{\mathrm{CO}} \approx 10^{21}$ than the empirical fit derived from the southern population. However, we see that although Figure 5 (middle panel) shows a robust compliance to the empirical line width-luminosity relationship, the sizeline width relationships (Figure 5, right panel) do not, and this implies the clouds in the north SMC are significantly relatively under-luminous compared to their southwestern counterparts.

Other quantitative indicators of differences in the northern and southern star formation environments exist; Davies et al. (1976) compiled a catalog of $\mathrm{H}$ II regions in the SMC that was later expanded by Bica et al. (2008). A more thorough study of the relationship of the northern and southern $\mathrm{H}$ II regions is deferred to a later study, however we conduct a Kolmogorov-Smirnov $(\mathrm{K}-\mathrm{S})$ test on the size of the $\mathrm{H}$ II regions in the northern and southern parts of the SMC (i.e., northern clouds are those that are west of $1.1^{\mathrm{hr}}$ and north of $-72^{\circ} 45^{\prime}$, while southern clouds are those west of $1.1^{\mathrm{hr}}$ and south of $-72^{\circ} 30^{\prime}$-approximately 

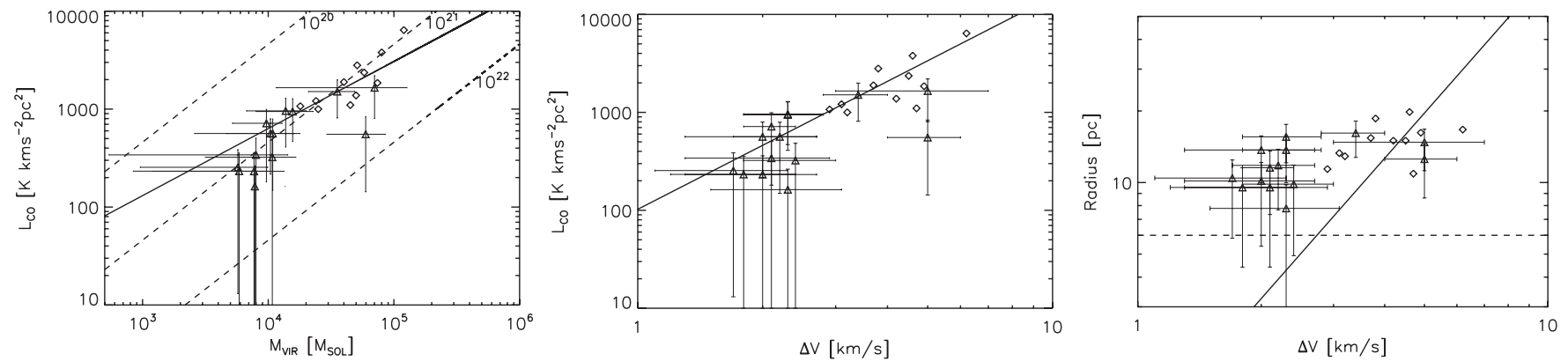

Figure 5. Comparisons of cloud properties measured from this study (triangles, with error bars), and those from the west of the SMC (SKP-III; diamonds; no errors cited, see Table 2 for estimations of errors of SKP-III data). Left panel: virial mass and luminosity, along with dotted lines of constant $X_{c o}=10^{20}, 10^{21}$, and $10^{22} \mathrm{~cm}^{-2}\left(\mathrm{~K} \mathrm{~km} \mathrm{~s}^{-1}\right)^{-1}$. Middle panel: line width and luminosity; and Right panel: line width and size, where the HPHW size of the Mopra beam ( $\left.\sim 6 \mathrm{pc}\right)$ is shown as a dotted line. The solid straight lines are empirically derived lines in SKP-III (no errors cited in the derivation of the empirical relationship).

the southern limit of the clouds examined by MAGMA-SMC) to test their morphological consistency. The K-S test shows that the northern population has a probability of $\sim 3 \%$ of being representative of the southern population, and we find the $\mathrm{H}$ II regions in the north are, on average, $60 \%$ larger than $\mathrm{H}$ in regions in the south, where the number populations of the north and south $\mathrm{H}_{\text {II }}$ data sets are 82 and 139, respectively. Generally, the sizes of the H II regions can be modulated by local density of pressure inhomogeneities, or are even just a result of differences in the mean ionizing luminosities and this is a further indicator that the two populations are evolving within different ambient conditions.

\subsection{The Compact Nature of $\mathrm{CO}$ in the Northern SMC}

To examine the compactness of the detected $\mathrm{CO}$ emission regions, we compare $\mathrm{CO}$ luminosities from the lower resolution and wider field NANTEN maps to the results presented here. Our intention is to check that Mopra has detected the same total flux as NANTEN over the same scale ranges, which will reveal the quantity of extended and diffuse CO. We are severely limited by the size of the field sampled by Mopra; the $5^{\prime} \times 5^{\prime}$ arcminute fields that have been measured in the Mopra survey subtend only two beams, or 2.5 pixels in the NANTEN data set.

Essentially, we are using the NANTEN data set as a control to check and compare the flux detected by the combination of the Mopra observations and CLOUDPROPS processing. We focus this test on the emission regions measured by Mopra and CLOUDPROPS only; we form an $I_{\mathrm{CO}}$ map using only spectra that have been identified by CLOUDPROPS as containing emission, thereby ignoring the noise-dominated parts of the map, which are instead set to zero, and then smooth the Mopra data set to the same resolution as NANTEN (2'.6). The NANTEN maps extend over a much wider field than the Mopra maps, and we further limit the size of the field used in the following test to radii $\lesssim 180$ pc (approximately 4 beams in the NANTEN data set, and 12 beams in the Mopra data set) to avoid confusion by other nearby $\mathrm{CO}$ complexes that are far outside the Mopra complexes, but that will contaminate the NANTEN data set through its wider beam.

We integrate the smoothed Mopra data set and the NANTEN data set over areas with incrementally increasing radii, focusing on each the complexes NE-1a, b, c; NE-3a, b, c, d, e, f, g; and NE-4a, b, c in their entirety: e.g., we simply treat the NE-4 complex as a whole and increase the scale of the test beyond the dimensions of the complex. Examining the whole of the complexes in this way ensures that we are considering entire complexes as observed by NANTEN (i.e., all the components of NE-1 are confused by the NANTEN beam). In general, we choose a point that is approximately central to each of the fields as our zero radius, although the exact position of the zero radius is generally unimportant.

We show in Figure 6 the $L_{\mathrm{CO}}(R) d r$ for NE-1, NE-3, and NE-4 from the NANTEN and the smoothed Mopra data. We find that the power measured by Mopra becomes equal to that measured by NANTEN in complexes NE-1 and NE-4 within the sampled scale ranges (Figure 6, left and right panels). The exact scale of the agreement is somewhat dependent on the distribution of the $\mathrm{CO}$ within the complex, but in both of these cases we find that Mopra measures the same total power as NANTEN within the $\sim 10^{\prime}$ fields. This result suggests that these the clouds (NE-1, NE-4) do not posses an extended $\mathrm{CO}$ envelope on radii much larger than those sampled in Figure 6 and listed in Table 1. The spatial range of this part of the study is limited to $\sim 10$ arcmin (where $10^{\prime}$ subtends $\sim 170 \mathrm{pc}$ at the $\sim 60 \mathrm{kpc}$ distance of SMC), and although these high-resolution observations do not completely eliminate the possibility of a weak and extended envelope, the important result is that the total flux measured on the small scales by Mopra is the same as that measured with the lower resolution and wider field NANTEN telescope.

The azimuthally integrated $L_{\mathrm{CO}}$ of NE-3, on the other hand, (middle panel of Figure 6) shows a great difference in the fluxes measured by Mopra and NANTEN. The figure shows a strong divergence of the two data sets at the approximate mean size of the Mopra maps, which suggests that the NANTEN data is being affected by a more extended envelope by a region to the east of NE-3 (see Figure 2). As much of this area is outside that observed by Mopra, it does not contribute to the Mopra data set. Until this region is fully sampled, we are not able to make a conclusion regarding the existence of any extended $\mathrm{CO}$ envelope that may be associated with NE-3.

\section{3. $\mathrm{CO}$ and $\mathrm{HI}$}

Wong et al. (2009) have made a thorough comparison of the properties of the $\mathrm{HI}$ and $\mathrm{CO}$ emission profiles throughout the LMC. Most notably, they found that $\mathrm{CO}$ is detected only toward relatively high $\mathrm{H}$ I column densities, where $N(\mathrm{HI}) \geqslant 10^{21} \mathrm{~cm}^{-1}$, and that these column densities are a necessary, but insufficient requirement for the formation of $\mathrm{CO}$ in the $\mathrm{LMC}$. The $\mathrm{H}$ I found in the LMC exists largely in single-component profiles where it is appropriate to assume that where the centroids of the $\mathrm{CO}$ and $\mathrm{H}$ I profiles coincide for a given line of sight, the two components are co-moving and co-evolving. In contrast, the neutral hydrogen component of the SMC is often complex in both position and in velocity: the $\mathrm{H}$ I profiles in the line of sight of the $\mathrm{CO}$ emission 

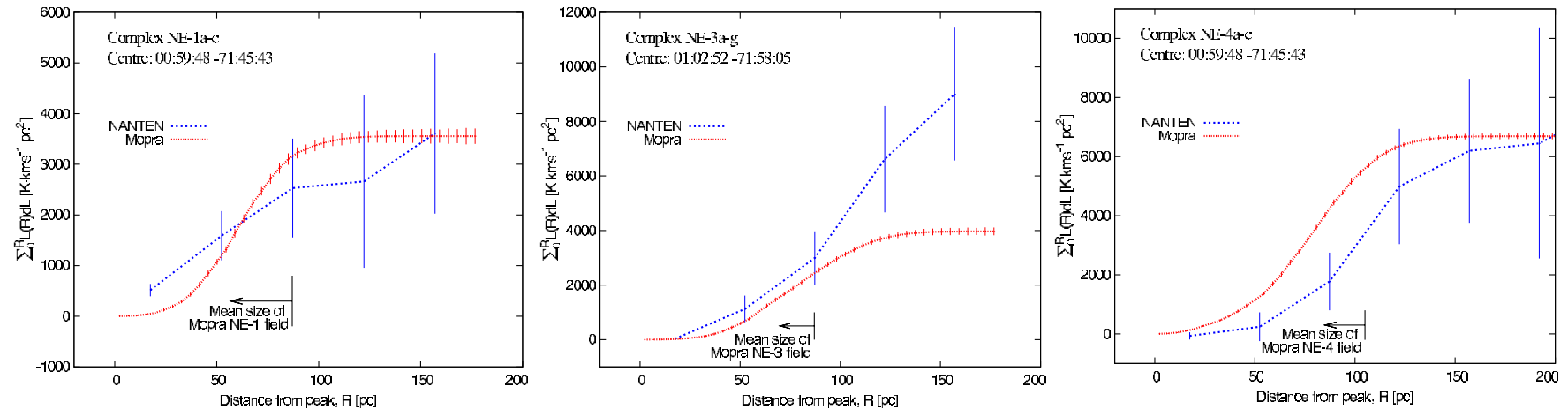

Figure 6. Azimuthally integrated brightness plots of NANTEN and (2'.6-smoothed) Mopra observations of complexes NE-1 (left panel), NE-3 (middle panel), and NE-4 (right panel). Error bars indicate the cumulative sum of the $1 \sigma$ noise level per pixel. Total power measured by Mopra for complexes NE-1 and NE- 4 equate with that of NANTEN after some scale, indicating that the same total power is measured over the total area (despite the larger beam size of NANTEN), arguing for an absence of any larger extended CO envelope. Complex NE-3 shows extended emission measured by NANTEN from additional CO components outside and to the east of the area observed by Mopra. Note that the geometry of the observed regions are generally highly irregular, the indicated radii is that of an equivalent-area circle and not the maximum extents of the test areas.

(A color version of this figure is available in the online journal.)

regions often contain a number of Gaussian components (see Figures 3 and 4), and it is inaccurate to assume that all the $\mathrm{HI}$ found along a particular sight is necessarily associated with the detected $\mathrm{CO}$ emission.

To isolate the $\mathrm{HI}$ component that is associated with the $\mathrm{CO}$, we assume that an $\mathrm{HI}$ and $\mathrm{CO}$ component must be co-moving, and constrain a multiple-component Gaussian fit with the CO emission velocity centroid as a fixed parameter. We do not assume that the $\mathrm{CO}$ and $\mathrm{H}$ I components are similarly turbulencebroadened (i.e., $\Delta V_{\mathrm{CO}} \neq \Delta V_{\mathrm{hi}}$ ).

For this analysis, we first smooth and re-grid the $\mathrm{CO}$ data set to the same geometry as the Hi data set (i.e., to a $98^{\prime \prime}$ beam, and $60^{\prime \prime}$ per pixel, which results in some dependence of adjacent pixels). As smoothing dilutes the peak brightness somewhat, we make this test only on the eight brightest CO clouds; NE-1a, c; NE-3a, c, g; and NE-4a, c $\mathrm{co}_{\mathrm{lo}} \mathrm{hi}$.

We found in all cases that fitting two Gaussian components provided a significant improvement in the summed reduced $\chi^{2}$ $\left(\sum \chi_{r}^{2}\right.$ ) parameter (by a factor of at least 2 , and a mean improvement by a factor of 4 , calculated where $T_{\mathrm{H}_{\mathrm{I}}}>3 \sigma_{\mathrm{H}_{\mathrm{I}}}$ ), but that fitting three components provided no further significant improvement. We have therefore fit two components to the $\mathrm{HI}$ profile and constrained one of them according to the measured CO centroid velocity, as listed in Table 1. In general, constraining one of the two $\mathrm{H}$ I components by the $\mathrm{CO}$ velocity centroids did not result in a large degradation of $\sum \chi_{r}^{2}$ compared to a completely unconstrained two-component fit: in the unconstrained case, the $\sum \chi_{r}^{2}$ was always of the order unity, while constraining the fit resulted in an average increase of the $\sum \chi_{r}^{2}$ by $20 \%$, although an increase by $40 \%$ was found for the most extreme cases. The largest $\sum \chi_{r}^{2}$ value was returned for fitting to cloud NE-3c $\left(\sum \chi_{r}^{2}=5.1\right)$ and the smallest $\sum \chi_{r}^{2}$ value was returned from fitting to cloud NE-4a $\left(\sum \chi_{r}^{2}=1.7\right)$. Note that in the case of NE-4, which contains two distinct H I profiles, fits were made only to the component within the velocity range of interest. For example, while fitting to the high-velocity component for NE-4c $\mathrm{c}_{\text {high }}$, the low-velocity $\mathrm{H}$ i component was ignored. Constraining one of the fitted $\mathrm{H}$ I velocity centroids by the $\mathrm{CO}$ component usually resulted in a significant (up to $100 \%$ ) change in the integrated $\mathrm{H}$ I from a completely unconstrained fit. Clouds $\mathrm{NE}-1 \mathrm{a}$ and NE-1c were the only examples where constraining the fit did not significantly change the measured integrated $\mathrm{H}$.

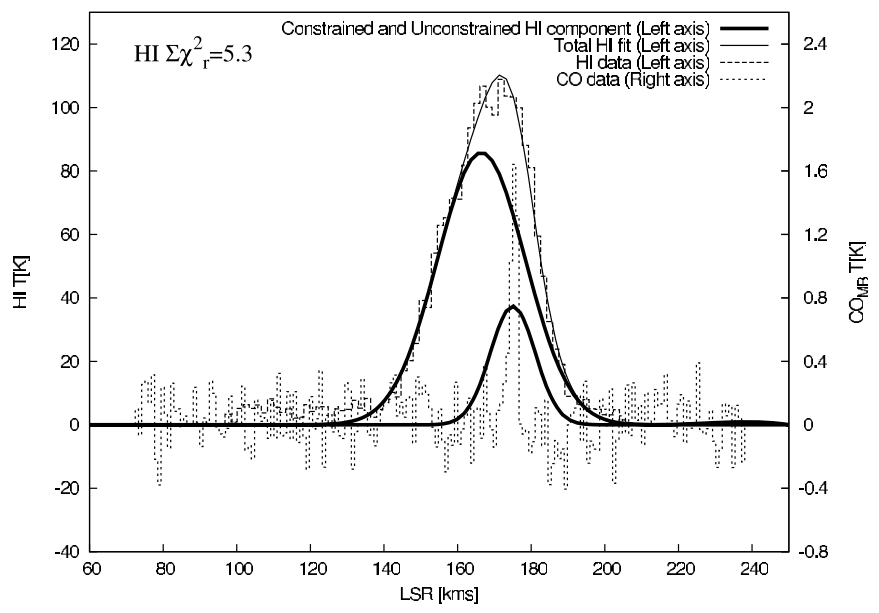

Figure 7. Two-component fitting made to $\mathrm{HI}$, where one component is constrained by the velocity centroid (marked with a short vertical line). The $\sum \chi_{r}^{2}$ parameter is shown, indicating a reasonable fit by constrained, twocomponent fit. The $\mathrm{H}$ I emission over $80-130 \mathrm{~km} \mathrm{~s}^{-1}$ is real, but is neither fitted nor included in the calculation of $\sum \chi_{r}^{2}$ (see the text for details). The heavy solid lines indicate the two fitted components. The lighter solid line is the total fitted result. The heavier dashed line shows the $\mathrm{H}$ I brightness temperature (left axis) and the lighter, noisier dashed line shows the CO brightness temperature (right axis).

An example of this process applied to cloud NE-3c is shown in Figure 7, the integrated intensity of the $\mathrm{HI}$ component associated with each $\mathrm{CO}$ velocity centroid is listed in Table 3, and plotted against $\mathrm{CO}$ integrated brightness in Figure 8. Figure 8 also shows data from the study of the LMC by Wong et al. (2009).

We do not have a means to presume velocity components to disentangle the complex $\mathrm{HI}$ profiles at positions where $\mathrm{CO}$ is not detected. As such, we are unable to make a robust statistical examination of the null case; i.e., the values of Integrated H I where $\mathrm{CO}$ is not detected. Figure 8 therefore presents only data points where both $\mathrm{CO}$ and $\mathrm{HI}$ are detected, and we find that the ranges of these two qualities are consistent with those in the LMC. As we have observed some of the brightest $\mathrm{CO}$ regions in the northern SMC, this suggests that the conclusions made by Wong et al. (2009) regarding the distribution of $\mathrm{CO}$ and $\mathrm{H}_{\mathrm{I}}$ in the LMC apply also to the SMC: that CO in the northern SMC 


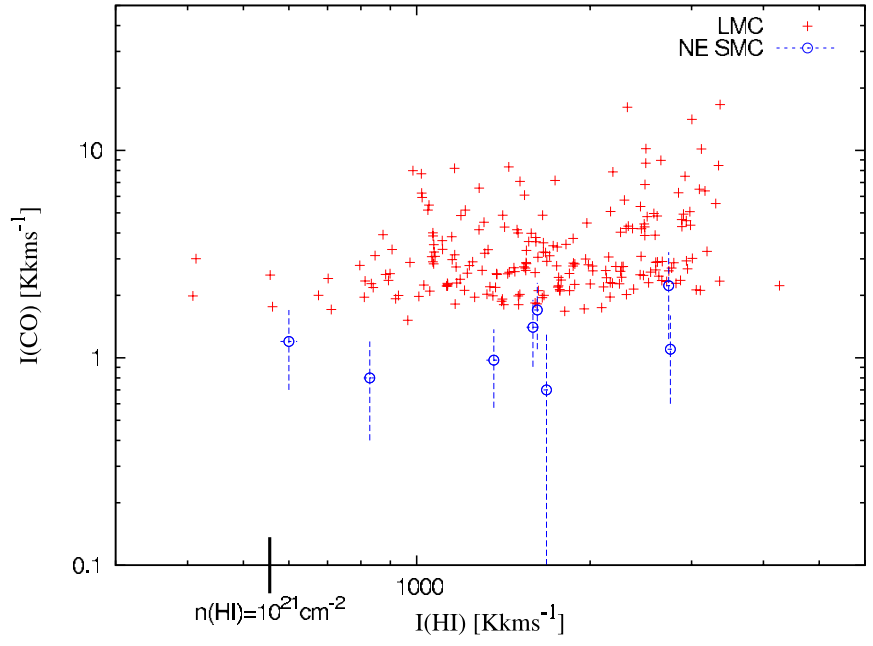

Figure 8. Plus signs: $I_{\mathrm{CO}} / I_{\mathrm{H}}$ of Molecular clouds in the LMC (Wong et al. 2009). Circles: $I_{\mathrm{CO}} / I_{\mathrm{H}_{\mathrm{I}}}$ of Molecular clouds in the SMC (this publication). CO clouds in the SMC are typically weaker than those in the LMC, and so the SMC data points plot below those of the LMC. Note that the SMC Mopra data are smoothed to the $98^{\prime \prime}$ resolution of the H I data set. An H I column density of $n \approx 10^{21} \mathrm{~cm}^{-2}$ is also indicated with a vertical line, to indicate the $n \mathrm{H} \mathrm{I}$ starforming threshold observed in the LMC by Luks \& Rohlfs (1992).

(A color version of this figure is available in the online journal.)

requires high $\mathrm{H}$ I column densities as a minimum condition for formation. Note that the plot shows that the integrated intensities of the CO clouds in the SMC are generally smaller (by a few factors) than those of the LMC, which is an already-anticipated result (e.g., Israel et al. 1993).

\subsection{Dust, $\mathrm{CO}$, and $\mathrm{HI}$}

Figures 2(b), (d), and 4(c) show the relative distribution of the detected $\mathrm{CO}$ against that of emission at $160 \mu \mathrm{m}$ (Bolatto et al. 2007) for NE-1, NE-3, and NE-4, respectively. We defer a more comprehensive analysis of the relationship of the smallscale $\mathrm{CO}$ to the $160 \mu \mathrm{m}$ emission to a later paper; however, we generally find from these new high-resolution data that the $\mathrm{CO}$ clouds in the more quiescent northern SMC are compact and are correlated only with the very brightest peaks of $160 \mu \mathrm{m}$ emission; i.e., bright $\mathrm{CO}$ occurs only with bright $160 \mu \mathrm{m}$ and $\mathrm{H} \mathrm{I}$ (e.g., the western CO peak in NE-3), and bright $160 \mu \mathrm{m}$ occurs without any detectable CO (e.g., the eastern $160 \mu \mathrm{m}$ peak in NE-3).

Variations in the dust-to-gas ratio, temperature variations of the dusty component, or photo-disassociation processes may account for inconsistencies in the correlations of the $160 \mu \mathrm{m}$ brightness and the $\mathrm{CO}$ integrated intensity but the point remains that the correlation of these emission data sets toward the northern SMC is not robust, emphasizing both the need for higher resolution $\mathrm{H}$ I observations, and also that estimates of the $X$ factor may be highly variable throughout the SMC (see also Leroy et al. 2007).

\subsection{Estimates of $X_{\mathrm{CO}}$}

Our high-resolution observations also allow us to make a new estimate of the SMC $X_{\mathrm{CO}}$ factor using virial masses estimated directly from the data. Using the same approach as Pineda et al. (2009); $X \sim M_{\mathrm{vir}} / L_{\mathrm{CO}}$ (and assuming a ratio the total gas mass to $\mathrm{H}_{2}$ gas mass of 1.36), we find a range of $X$ factor values of $5.4 \times 10^{20}-4.3 \times 10^{21} \mathrm{~cm}^{-2}\left(\mathrm{~K} \mathrm{~km} \mathrm{~s}^{-1}\right)^{-1}$, with a mean value of $1.3_{-0.8}^{+1.6} \times 10^{21} \mathrm{~cm}^{-2}\left(\mathrm{~K} \mathrm{~km} \mathrm{~s}^{-1}\right)^{-1}$ for the northern
Table 3

$I(\mathrm{CO})$ and $I(\mathrm{HI})$ of Northern CO Cloud Population

\begin{tabular}{lcc}
\hline \hline Cloud & \multicolumn{1}{c}{$\begin{array}{c}I_{\mathrm{H}_{\mathrm{I}}} \\
\left(\mathrm{K} \mathrm{km} \mathrm{s}^{-1}\right) \times 10^{3}\end{array}$} & $\begin{array}{c}I_{\mathrm{CO}} \\
\left(\mathrm{K} \mathrm{km} \mathrm{s}^{-1}\right)\end{array}$ \\
\hline $\mathrm{NE}-1 \mathrm{a}$ & $1.62 \pm 0.02$ & $1.7 \pm 0.6$ \\
$\mathrm{NE}-1 \mathrm{c}$ & $0.830 \pm 0.02$ & $0.8 \pm 0.4$ \\
$\mathrm{NE}-3 \mathrm{a}$ & $1.68 \pm 0.02$ & $0.7 \pm 0.6$ \\
$\mathrm{NE}-3 \mathrm{c}$ & $0.600 \pm 0.02$ & $1.2 \pm 0.5$ \\
$\mathrm{NE}-3 \mathrm{f}$ & $2.76 \pm 0.03$ & $1.1 \pm 0.5$ \\
$\mathrm{NE}-4 \mathrm{a}$ & $1.59 \pm 0.04$ & $1.4 \pm 0.5$ \\
$\mathrm{NE}-4 \mathrm{c}_{\text {hi }}$ & $1.36 \pm 0.04$ & $0.9 \pm 0.4$ \\
$\mathrm{NE}-4 c_{\text {low }}$ & $2.74 \pm 0.03$ & $2.2 \pm 1$ \\
\hline
\end{tabular}

SMC cloud population. The errors of these measurements are high due to the poor $\mathrm{S} / \mathrm{N}$ and matching of the beam and cloud radii (as discussed in Section 3). Note that this value is approximately a factor of 4 higher than that found for the LMC (Pineda et al. 2009; after adjusting for their slightly different definition of $M_{\mathrm{vir}}$ ). Given that the ensemble virial masses (i.e., the velocity dispersions and radii) of the northern SMC population is approximately represented by the smallest members of the LMC cloud population (Pineda et al. 2009), this suggests that the SMC harbors a significantly under-luminous $\mathrm{CO}$ cloud population.

The $X_{\mathrm{SMC}}$ factor was also estimated by Rubio et al. (1991) from low-resolution observations of the same northern population. Later, $X_{\mathrm{SMC}}$ factor estimates were made from higher resolution observations of the southwest population in SKP-III. These two studies used different methods to estimate the $X_{\text {SMC }}$ factor. (1) The earlier paper assumes that the size-line width relationship of CO clouds in the Galaxy and SMC is consistent, and they estimated that SMC CO clouds were under-luminous compared to the Galaxy by a factor of 20 . The $X_{\text {SMC }}$ was therefore estimated to be $\sim 6 \times 10^{21} \mathrm{~cm}^{-2}\left(\mathrm{~K} \mathrm{~km} \mathrm{~s}^{-1}\right)^{-1}$; approximately 20 times higher than $X_{\mathrm{Gal}}$ (using the values for $X_{\mathrm{Gal}}$ at the time of their writing). (2) The later work by the SEST key projects using $\sim 45^{\prime \prime}$ resolution observations of clouds in the southwest of the SMC made estimates of the $X_{\mathrm{SMC}}$ for each cloud by assuming a specific fractional mass of the molecular hydrogen. The resulting $X_{\mathrm{SMC}}$ values range between $2.5 \times 10^{20} \mathrm{~cm}^{-2}(\mathrm{~K}$ $\left.\mathrm{km} \mathrm{s}^{-1}\right)^{-1}$ and $2.6 \times 10^{22} \mathrm{~cm}^{-2}\left(\mathrm{~K} \mathrm{~km} \mathrm{~s}^{-1}\right)^{-1}$, with stronger clustering at approximately $10^{21} \mathrm{~cm}^{-2}\left(\mathrm{~K} \mathrm{~km} \mathrm{~s}^{-1}\right)^{-1}$. No errors were estimated for these values.

The issue of the relative distributions of $\mathrm{CO}$ and $\mathrm{H}_{2}$ was discussed in some detail by Leroy et al. (2007), who used the 2.6 FWHM NANTEN data to show that the CO was more compact than the $\mathrm{H}_{2}$ (determined from $160 \mu \mathrm{m}$ ) by a factor of approximately 1.3 . They also find that the $X_{\mathrm{SMC}}$ factor varies throughout the SMC by a factor of $\sim 2$, however, using a factor of 1.3 as a parameter to adjust for the relative sizes of the $\mathrm{H}_{2}$ and $\mathrm{CO}$ emission regions, they estimate a mean $X_{\mathrm{SMC}}$ factor of $6 \times$ $10^{21} \mathrm{~cm}^{-2}\left(\mathrm{~K} \mathrm{~km} \mathrm{~s}^{-1}\right)^{-1}$. We find from our higher resolution data that the average radius of the clouds in the SMC is approximately $12 \mathrm{pc}$, which would result in a correction factor of approximately 3.3 and would adjust their FIR-based estimate of the $X_{\text {SMC }}$ to $2.4 \times 10^{21} \mathrm{~cm}^{-2}\left(\mathrm{~K} \mathrm{~km} \mathrm{~s}^{-1}\right)^{-1}$. This value is consistent with COderived virial masses derived earlier. Leroy et al. (2007) indicate that the difference between the mm-derived virial masses and the $\mu \mathrm{m}$-derived masses, which would indicate instability and would otherwise lead to cloud fragmentation, may be balanced by an ambient pervasive magnetic field. The small correction found from more exact cloud radii measurements presented here 
reduces the necessary strength of any such field (see also Bot et al. 2007).

\section{SUMMARY}

We have produced the highest resolution observations of the molecular cloud population in the northern part of the SMC, to a sensitivity of $210 \mathrm{mK}$ per $0.9 \mathrm{~km} \mathrm{~s}^{-1}$ channel and resolving each cloud into 3-7 smaller clouds. We find that this northern population conforms to empirical luminosity-virial mass relationships found for the southern SMC population, yet they are generally much less luminous for their radius. Extrapolating the empirical size-line width relationship derived for the southern clouds implies that the northern population has minimum radii of a 4 pc. A comparison with wider field $\mathrm{CO}$ data suggests that two of the three cloud complexes do not exist in a large-scale and weak $\mathrm{CO}$ envelope, and that highresolution $\mathrm{H}$ I data are imperative for more clearly understanding the distribution of molecular material within the SMC. We find that, as for the LMC, CO in the SMC occurs only with high (i.e., $\left.>10^{21} \mathrm{~cm}^{-1}\right) \mathrm{H}$ I column density, which suggests that as for the LMC, such column densities are required for its formation. We use these molecular data to estimate $X_{\text {SMC }}$ factors to be approximately $1.3 \times 10^{21}$, and our high-resolution data allow us to apply a more appropriate correction factor to previous estimates of the $X_{\mathrm{SMC}}$ from $\mu \mathrm{m}+\mathrm{H}$ I data, and arrive at roughly consistent values.

All astronomical images used in this publication were made with the use of the KARMA package (Gooch 1997). J.L.P. was supported by an appointment to the NASA Postdoctoral Program at the Jet Propulsion Laboratory, California Institute of Technology, administered by Oak Ridge Associated Universities through a contract with NASA. M.R. wishes to acknowledge support from FONDECYT (CHILE) grant No. 1080335. M.R. is supported by the Chilean Center for Astrophysics FONDAP No. 15010003

\section{REFERENCES}

Bica, E., Bonatto, C., Dutra, C. M., \& Santos, J. F. C. 2008, MNRAS, 389, 678 Blitz, L., Fukui, Y., Kawamura, A., Leroy, A., Mizuno, N., \& Rosolowsky, E. 2007, in Protostars and Planets V, Giant Molecular Clouds in Local Group Galaxies, ed. B. Reipurth, D. Jewitt, \& K. Keil (Tucson, AZ: Univ. Arizona Press), 81

Bolatto, A. D., Leroy, A. K., Rosolowsky, E., Walter, F., \& Blitz, L. 2008, ApJ, 686, 948

Bolatto, A. D., et al. 2007, ApJ, 655, 212

Bot, C., Boulanger, F., Rubio, M., \& Rantakyro, F. 2007, A\&A, 471, 103

Cioni, M.-R. L., van der Marel, R. P., Loup, C., \& Habing, H. J. 2000, A\&A 359,601

Davies, R. D., Elliott, K. H., \& Meaburn, J. 1976, Mem. R. Astron. Soc., 81, 89

Gooch, R. E. 1997, PASA, 14, 106

Hughes, A., et al. 2010, MNRAS, submitted

Israel, F. P., et al. 1993, A\&A, 276, 25

Israel, F. P., et al. 2003, A\&A, 406, 817

Ladd, N., Purcell, C., Wong, T., \& Robertson, S. 2005, PASA, 22, 62

Landman, D. A., Roussel-Dupre, R., \& Tanigawa, G. 1982, ApJ, 261, 732

Larsen, S. S., Clausen, J. V., \& Storm, J. 2000, A\&A, 364, 455

Leroy, A., Bolatto, A., Stanimirovic, S., Mizuno, N., Israel, F., \& Bot, C. 2007, ApJ, 658, 1027

Luks, T., \& Rohlfs, K. 1992, A\&A, 263, 41

Maloney, P., \& Black, J. H. 1988, ApJ, 325, 389

McKee, C. F. 1989, ApJ, 345, 782

Mizuno, A., Yamaguchi, R., Tachihara, K., Toyoda, S., Aoyama, H., Yamamoto, H., Onishi, T., \& Fukui, Y. 2001, PASJ, 53, 1071

Ott, J., et al. 2008, PASA, 25, 129

Pak, S., Jaffe, D. T., van Dishoeck, E. F., Johansson, L. E. B., \& Booth, R. S. 1998, ApJ, 498, 735

Pineda, J. L., Ott, J., Klein, U., Wong, T., Muller, E., \& Hughes, A. 2009, ApJ, 703,736

Rolleston, W. R. J., Trundle, C., \& Dufton, P. L. 2002, A\&A, 396, 53

Rolleston, W. R. J., Venn, K., Tolstoy, E., \& Dufton, P. L. 2003, A\&A, 400, 21

Rosolowsky, E., \& Leroy, A. 2006, PASP, 118, 590

Rubio, M., Contursi, A., Lequeux, J., Probst, R., Barbá, R., Boulanger, F., Cesarsky, D., \& Maoli, R. 2000, A\&A, 359, 1139

Rubio, M., Garay, G., Montani, J., \& Thaddeus, P. 1991, ApJ, 368, 173

Rubio, M., Lequeux, J., \& Boulanger, F. 1993a, A\&A, 271, 9

Rubio, M., et al. 1993b, A\&A, 271, 1

Rubio, M., et al. 1996, A\&AS, 118, 263

Stanimirovic, S. 1999, PhD thesis, Univ. Western Sydney

Wong, T., et al. 2009, ApJ, 696, 370 